\title{
BOUNDED FUNCTIONS AND FOURIER TRANSFORMS
}

\author{
R. E. EDWARDS
}

1. Statement of the principal result. The class of sequences obtainable as Fourier coefficients of measures on the circle group presents numerous structure problems of interest. Amongst the earliest results somewhat akin to that stated below appear Banach's theorems about lacunary coefficients; see e.g., [4, pp. 215-220]. More recently Helson [3] has studied analogous questions when the group concerned is the real line.

The aim of the present paper is to give a simple proof by abstract methods of the following

THEOREM. Let $X$ be a compact abelian group with dual group $Y$, and let $S$ be a subset of $Y$. In order that each bounded function on $S$ shall there coincide with the Fourier transform of some Radon measure on $X$, it is already sufficient that the following condition be fulfilled:

(C) The characteristic function of each subset of $S$ is equal on $S$ to the transform of some Radon measure on $X$.

The method of proof uses only the most elementary properties of the Fourier transformation and, for precisely this reason, adapts itself easily to several other transformations acting on measures on semigroups. The remaining tools are certain basic ones now fairly familiar in functional analysis, namely: (1) the Bipolar Theorem $[2$, p. 52 , Proposition 3; Corollaire 2, p. 67 ]; and (2) the Baire Category Theorem and its consequences, in particular the fact that every Banach space has the property of being "tonnelé," as M. Bourbaki would say ([2, p. 2, Corollaire ]; cf. also the analogous Théorème 11, p. 19, of [1]). This last signifies merely that every lower semicontinuous seminorm on a Banach space is necessarily continuous.

It is a pleasure to record my thanks to Professor Edwin Hewitt for his several comments, most of which have been incorporated with advantage. To him is due a major improvement, namely the removal of an initial restriction of countability on $S$ which was imposed (unnecessarily) by my original proof of Proposition 2 blow.

2. Preliminary propositions. $X$ and $Y$ shall be locally compact abelian groups in duality, and $(x, y)$ shall denote the character function. The Fourier transform $\hat{f}$ of a function or a measure $f$ on $X$ is

Received by the editors September 15, 1956. 
defined in the usual manner, viz. ${ }^{1}$

$$
\hat{f}(y)=\int_{X}(x, y)^{*} \cdot f(x) d x \text { or } \quad \int_{X}(x, y)^{*} \cdot d f(x)
$$

for $y$ in $Y, d x$ denoting the Haar measure on $X$.

The first proposition is more general than is necessary, but it probably has some intrinsic interest. In it figures a positive Radon measure $\sigma$ on $Y$. A function $h$ is said to be "locally integrable" for $\sigma$, if it is integrable for $\sigma$ over each compact set in $Y$.

Proposition 1. Let $\sigma$ be a positive Radon measure on $Y$. If a function $h$, locally integrable for $\sigma$, is such that

$$
\int|h \cdot \hat{f}| d \sigma<+\infty \quad\left(f \in L^{1}(X)\right)
$$

then indeed $h$ is integrable for $\sigma$. Furthermore, there exists a positive number $c$, independent of $h$, such that

$$
\int|h| d \sigma \leqq c \cdot \operatorname{Sup}_{\|f\|_{1} \leqq 1} \int|h \cdot \hat{f}| d \sigma
$$

for all h satisfying (1).

Proof. For the first part, consider the function

$$
P: f \rightarrow \int|h \cdot \hat{f}| d \sigma
$$

with domain $L^{1}(X)$ (the usual Lebesgue space constructed relative to Haar measure on $X) . P$ is a finite-valued by hypothesis, and it is clearly a seminorm on $L^{1}(X)$. Since also

$$
P(f)=\operatorname{Sup}_{\boldsymbol{K}} \int_{K}|h \cdot \hat{f}| d \sigma,
$$

where $K$ ranges over compact subsets of $Y$, it appears at once that $P$ is lower semicontinuous. By the result quoted in $\$ 1, P$ is therefore continuous. In other words, there is a positive number $p$, independent of $f$, such that

$$
P(f) \leqq p \cdot\|f\|_{1} \equiv p \cdot \int_{X}|f(x)| d x .
$$

$1 *$ Denotes complex conjugate. 
If we now allow $f$ to range over an "approximate identity" in $L^{1}(X)$, it follows that

$$
\int_{K}|h| d \sigma \leqq p
$$

for all compacts $K$ in $Y$, whence follows the first assertion.

For the second part, introduce the set $\Lambda$ of $h$, locally integrable for $\sigma$, which satisfy (1). $\Lambda$ is plainly a vector space which, as we have just seen, may be identified with $L^{1}(\sigma)$ when topologies are neglected. It follows incidentally that

$$
N(h) \equiv \operatorname{Sup}_{\|\|_{1} \leqq 1} \int|h \cdot \hat{f}| d \sigma
$$

is finite on $\Lambda$, being at most equal to $\int|h| d \sigma$. Plainly, $N$ is a norm on $\Lambda$.

The next step is to show that $\Lambda$ is complete for $N$. Suppose that $\left(h_{n}\right)$ is a sequence in $\Lambda$ such that $N\left(h_{m}-h_{n}\right) \rightarrow 0$ as $m, n \rightarrow \infty$. The sequence $\left(h_{n}\right)$ is then Cauchy in the space $L^{1}(K, \sigma)$ for each compact $K$ in $Y$, so it follows that there is a function $h$, locally integrable for $\sigma$, such that $h_{n} \rightarrow h$ in $L^{1}(K, \sigma)$ for each $K$. Consequently

$$
\int_{K}|h \cdot \hat{f}| d \sigma=\lim _{n \rightarrow \infty} \int_{K}\left|h_{n} \cdot \hat{f}\right| d \sigma \leqq \limsup _{n \rightarrow \infty} N\left(h_{n}\right)
$$

is finite for each $K$ and bounded with respect to $K$. This implies that $h$ satisfies (1) and thus belongs to $\Lambda$, the last inequalities holding uniformly for $\|f\|_{1} \leqq 1$. Next, given $\epsilon>0$, we can choose an integer $n_{0}$ $=n_{0}(\epsilon)$ so that

$$
\int\left|h_{m}-h_{n}\right| \cdot|\hat{f}| d \sigma \leqq \epsilon
$$

provided only that $m, n \geqq n_{0}$ and $\|f\|_{1} \leqq 1$. The same holds a fortiori when the integration is restricted to a compact $K$; when this is done, and one then allows $m$ to tend to infinity, it follows that

$$
\int_{K}\left|h-h_{n}\right| \cdot|\hat{f}| d \sigma \leqq \epsilon
$$

for all $K$, all $n \geqq n_{0}$, and all $f$ satisfying $\|f\|_{1} \leqq 1$. In this, first allow $K$ to expand, the result being that

$$
\int\left|h-h_{n}\right| \cdot|\hat{f}| d \sigma \leqq \epsilon
$$


for $n \geqq n_{0}$ and $\|f\|_{1} \leqq 1$. This signifies that $N\left(h-h_{n}\right) \leqq \epsilon$ for $n \geqq n_{0}$, i.e., that $\left(h_{n}\right)$ converges to $h$ in $\Lambda$. The completeness of $\Lambda$ is thus demonstrated.

Finally,

$$
\int|h| d \sigma=\operatorname{Sup}_{K} \int_{K}|h| d \sigma
$$

is a finite, lower semicontinuous seminorm on $\Lambda$ (equipped with $N$ ). A second application of the result stated in $\$ 1$ shows that this seminorm is continuous, and (2) is proved. As Professor Hewitt pointed out, once completeness of $\Lambda$ (for $N$ ) is established, one can make direct appeal to $[1$, p. 41 , Théorème 5$]$ in order to establish (2).

REMARKs. It makes no difference whether $h$ is real- or complexvalued. In the former case, we can everywhere restrict $f$ to satisfy $f(-x)=(f(x)) *$ identically, so that $\hat{f}$ is real-valued. This restriction, pointless here, simplifies subsequent arguments.

Proposition 2. Let $X$ be a compact abelian group, and let $S$ be a subset of the dual group $Y$. Suppose that condition (C) is satisfied. There exists a positive number $m$ with the property that the characteristic function of each subset of $S$ coincides on $S$ with the transform of some Radon measure on $X$ having total mass at most $m$.

Proof. Let $\Phi$ denote the set of functions on $S$ which are characteristic functions of subsets thereof. Equip $\Phi$ with the topology of simple convergence on $S$. The functions in $\Phi$ being precisely those taking only the values 0 or 1 , Tychonoff's theorem shows that $\Phi$ is compact. Hence condition (C), coupled with the Category Theorem for compact spaces, implies the existence of a positive number $m_{0}$, a $\phi_{0}$ in $\Phi$, and a finite set $S_{0} \subset S$, with this property: each $\phi$ in $\Phi$ which coincides on $S_{0}$ with $\phi_{0}$ is the limit in $\Phi$ of functions $\hat{\mu} \mid S$, where $\mu$ is a variable measure of total mass at most $m_{0}$. On the other hand, the set of such measures is weakly compact (qua subset of the dual of $C(X)$, the usual Banach space of continuous functions on $X$ ), whilst the mapping $\mu \rightarrow \widehat{\mu} \mid S$ is continuous for this weak topology and the topology of simple convergence on $S$. So it appears that each $\phi$ in $\Phi$ which coincides on $S_{0}$ with $\phi_{0}$ has the form $\hat{\mu} \mid S$, where $\mu$ has total mass at most $m_{0}$.

The remaining step is almost trivial. Enumerate $S_{0}$ as $\left(y_{j}\right)_{1 \leq j \leq k}$, and let $\phi_{j}$ be the characteristic function of the one-point set $\left\{y_{j}\right\}$. Any $\psi$ in $\Phi$ can be written

$$
\psi=\sum_{1 \leq j \leq k}\left(\psi\left(y_{j}\right)-\phi_{0}\left(y_{j}\right)\right) \cdot \phi_{j}+\phi
$$


where $\phi$ is by definition equal to $\phi_{0}$ on $S_{0}$ and to $\psi$ on $S-S_{0}$. By hypothesis (or as may be seen directly) each of the finitely many $\phi_{j}$ is a transform, and it appears that $\psi$ itself has the form $\hat{\mu} \mid S$, the total mass of $\mu$ being at most $k+m_{0}=m$. This completes the proof.

REMARK. There is of course a similar result concerning functions $\phi$ on $S$ taking values in any fixed finite set.

3. Proof of the theorem. We shall use (2) in the "real" form; see the Remark following the proof of Proposition 1. For $\sigma$ one chooses any positive measure with support precisely $S$.

Let $u$ be any real-valued function on $S$ satisfying $|u| \leqq 1$. By (2), any $h$ integrable for $\sigma$ satisfies

$$
\left|\int h u d \sigma\right| \leqq \int|h| d \sigma \leqq c \cdot \operatorname{Sup}_{\|f\|_{1} \leqq_{1}} \int|h \hat{f}| d \sigma .
$$

One may write here

$$
|h \hat{f}|=h \hat{f} \theta \text {, }
$$

where $\theta$ takes only the values \pm 1 on $S$. With the notation used in the proof of Proposition 2, any such $\theta$ can be written as $\phi_{1}-\phi_{2}$ with $\phi_{1}$ and $\phi_{2}$ in $\Phi$. Accordingly, by condition (C), one may also write $\theta=\hat{\mu}$ on $S$, where $\mu$ has total mass at most $2 m$. Then $\hat{f} \theta=(f * \mu)^{-}$on $S$, the measure $f * \mu$ again having total mass at most $2 m$. Thus finally

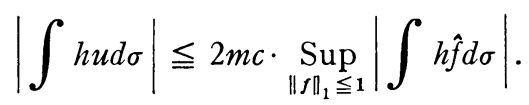

This system of inequalities, with $h$ ranging over functions integrable for $\sigma$, together with the Bipolar Theorem, shows that $u$ is adherent, weakly in $L^{\infty}(\sigma)$, to the set of transforms $\hat{f}$ with $\|f\|_{1} \leqq 2 m c$. Since $S$ is discrete, this implies a fortiori that $u$ is adherent, for the topology of simple convergence on $S$, to the set of functions $\hat{\mu} \mid S$ with $\mu$ a measure having total mass at most $2 m c$. However, exactly as in the proof of Proposition 2, one sees that this latter set is closed for the topology of simple convergence on $S$. From this follows the desired result.

4. The case in which $X$ is noncompact. If $X$ is noncompact, the arguments used in the proof of Proposition 2 and again in $\S 3$ are no longer valid. The principal difficulty is that, whilst the normbounded sets of measures remain relatively compact (for the weak topology of the dual of $C_{0}(X)$, the Banach space of continuous functions on $X$ which vanish at infinity), the weak convergence of a measure no longer implies the pointwise convergence of its trans- 
form. Indeed, it may be shown that weak convergence of a normbounded directed family of measures is equivalent to the convergence, weakly in $L^{\infty}(Y)$, of the transforms. This latter mode of convergence is much too coarse to respect discrete subsets $S$ of $Y$. Thus the statement of the theorem remains unverified.

Nevertheless, it is perhaps worth recording that the statement and the proofs are reinstated if at all stages one uses only measures selected from a suitable subset. Nothing very neat and decisive can be offered at present, so only the outlines will be sketched.

To begin with, Proposition 1 holds if therein $f$ is restricted further to have its support within any pre-assigned neighbourhood of 0 in $X$. No change in the proof is required.

Instead of taking all bounded Radon measures on $X$, select a subset $M$. Conditions may be imposed upon $M$ in order that the arguments of the proof of Proposition 2 and of $\$ 3$ remain valid. A reasonably minimal set of conditions is as follows: $M$ is the union of a countable increasing directed family $M_{m}(m=1,2, \cdots)$ of subsets, each $M_{m}$ being convex and circled; for each $m$ and each number $r>0$, there exists an $n$ such that $r \cdot M_{m} \subset M_{n}$; finally it is supposed that

(a) each $M_{m}$ is compact for some separated topology relative to which the functions $\mu \rightarrow \hat{\mu}(y)(y \in S)$ are continuous;

(b) for each $m$ there is a neighborhood $W_{m}$ of 0 in $X$ and an $n$ such that $M_{m}$ is mapped into $M_{n}$ by convolution with functions $f$ in $L^{1}(X)$ having support in $W_{m}$ and norm at most 1 .

In case $X$ is compact, $M_{m}$ has hitherto been taken simply as the set of all measures of total mass at most $m$, the topology in (a) as the weak topology, and $W_{m}=X$.

Here is an example illustrating the possibilities. Choose a sequence $\left(w_{p}\right)_{p \geqq 1}$ of "weight functions" on $X$, each of which will be assumed to be strictly positive and lower semicontinuous. Assume too that

$$
\lim _{x \rightarrow \infty} w_{p}(x)=+\infty
$$

whilst

$$
\operatorname{Sup}_{x^{\prime} \in X} w_{p}\left(x+x^{\prime}\right) / w_{p}\left(x^{\prime}\right)
$$

is locally bounded ( $q u \hat{a}$ function of $x$ ) for each $p$. For each pair $(p, q)$ of positive integers, let $M_{p, q}$ be the set of (necessarily bounded) Radon measures $\mu$ on $X$ for which

$$
\int w_{p} d|\mu| \leqq q
$$


where $|\mu|$ denotes the total variation of $\mu$. The $M_{m}$ will then be the double sequence $\left(M_{p, q}\right)$ after arbitrary enumeration has been effected.

It is easily verified that each $M_{p, q}$ is norm-bounded and weakly closed in the set of all bounded measures, hence is weakly compact: for this we use $\left(\mathrm{a}^{\prime}\right)$. But $\left(\mathrm{a}^{\prime}\right)$ also shows that if a variable $\mu$ in $M_{p, q}$ converges weakly, then its transform converges simply on $Y$; this is because $\left(\mathrm{a}^{\prime}\right)$ ensures that the measures in $M_{p, q}$ are, so to speak, "equivanishing at infinity." Thus (a) will hold. On the other hand, $\left(b^{\prime}\right)$ will ensure that (b) is satisfied.

Consequently the main theorem of $\$ 1$ will remain true, if "measure" is everywhere understood to mean "measure belonging to $M$," where $M=\mathrm{U}_{p, q \geq 1} M_{p, q}$. Indeed, what is more striking, if (C) holds with this interpretation of "measure," then in fact there is a pair $(p, q)$ such that every function $u$ on $S$ satisfying $|u| \leqq 1$ has the form $\hat{\mu} \mid S$ for some $\mu$ in $M_{p, q}$.

It will be noted that, if $X$ is the real line, and if the $w_{p}$ grow sufficiently rapidly, the measures involved will have transforms which are holomorphic in horizontal strips containing the real axis, or even entire functions.

\section{REFERENCES}

1. S. Banach, Théorie des opérations linéaires, Warszawa, 1932.

2. N. Bourbaki, Espaces vectoriels topologiques, Chap. III-V, Actualités Scientifiques et Industrielles, no. 1229, Paris, Hermann, 1955.

3. H. Helson, Fourier transforms on perfect sets, Studia Mathematica vol. 14 (1954) pp. 209-213.

4. A. Zygmund, Trigonometrical series, Warszawa-Lwow, 1935.

Birkbeck College, London, England 